Mini-Review

\title{
Inhibiting the Inhibitors, PTP1B as a Therapeutic Target in Myocardial Infarction
}

\author{
Lorna R. Fiedler, PhD* \\ Clinical Laboratory Sciences, Radcliffe Department of Medicine, MRCWeatherall Institute for Molecular Medicine, John Radcliffe Hospital, Headington, Oxford, OX3 \\ 9DS, UK
}

\section{"Corresponding author}

Lorna R. Fiedler, PhD

Clinical Laboratory Sciences, Radcliffe Department of Medicine, MRCWeatherall Institute for Molecular Medicine, John Radcliffe Hospital, Headington, Oxford, OX3 9DS, UK; E-mail: lorna.fiedler@gmail.com

\section{Article information}

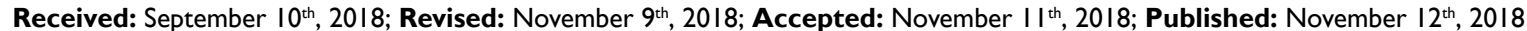

\section{Cite this article}

Fiedler LR. Inhibiting the inhibitors, PTPIB as a therapeutic target in myocardial infarction. Heart Res Open J. 2018; 5(I): 8-II. doi: I0.17I40/HROJ-5-I47

\begin{abstract}
Biological systems self-regulate through cycles of activation and inactivation, the balance of which is critical in permitting or suppressing signaling cascades and their downstream consequences. Kinase signaling pathways are regulated through phosphorylation (activation) and dephosphorylation (inactivation) events. Hyperactivity of kinase pathways plays a causal and critical role in the development of cardiac pathologies, and as such, the development of pharmacological inhibitors has been an intense area of investigation. Conversely, enhancing the activity of suppressed, but potentially beneficial kinase activities present an alternative therapeutic avenue. In this context, kinase dephosphorylation by phosphatases results in inactivation and suppression of the pathway. Thus inhibiting the inhibitors provides a method by which to enhance the activity of selected pathways. The protein tyrosine phosphatase 1B (PTP1B) has been implicated as a therapeutic target in several diseases but was considered to be undruggable. More recent development of inhibitors with improved specificity and pharmacological properties along with identification of novel indications has sparked renewed interest. This mini-review summarises the current status of PTP1B inhibitors in clinical trials and in pre-clinical models for new indications; myocardial infarction and heart failure.
\end{abstract}

\section{Keywords}

Myocardial infarction; Phosphatase inhibitors; PTP1B.

\section{Abbreviations}

HCM: Hypertrophic Cardiomyopathy; LDLR: Low-Density Lipoprotein Receptor; MI: Myocardial Infarction; PTP1B: Protein Tyrosine Phosphatase 1B.

\section{KINASE REGULATION}

$\mathrm{P}$ rotein kinase activities are regulated at multiple levels from transcription e.g. mRNA stability and splicing, to translational control and protein turnover through degradation. At the level of the protein itself, phosphorylation represents a major regulatory event that is mediated by protein kinases or receptor tyrosine kinases. Kinases are enzymes that catalyze phosphorylation of serine/threonine or tyrosine residues. Kinase activation itself is achieved through phosphorylation of key residues in the active site of the enzyme either by autoactivation through conformational changes, e.g. induced by ligand-receptor tyrosine kinase engage- ment, and/or phosphorylation by other kinases.

Phosphorylation outside of the active site region also provides binding sites for downstream targets and transmission of the activating signal downstream. Additionally, phosphorylation events can alter localization, providing a means of spatial regulation. In the context of cardiovascular disease (and indeed, every system and disease of the body), kinases have been shown to mediate a multitude of pathways that can result in deleterious outcomes. ${ }^{1}$ Thus there are many kinase candidate targets for pharmacological intervention in the clinic. 
Figure I: Phosphorylation is a Major Regulatory Event That Occurs Post-Translationally. Kinases Are Enzymes That Catalyse Phosphorylation of Serine/Threonine or Tyrosine Residues. Kinase Activation Itself is Achieved Through Phosphorylation of Key Residues in the Active Site of the Enzyme Either by Phosphorylation by Other Kinases (A) or by Auto Activation Through Conformational Changes, E.G. Induced by Ligand-Receptor Tyrosine Kinase Engagement (B). Phosphatases Keep Activity of Kinases in Check by Catalysing Removal of Critical Phosphate Groups to Dampen Activity, Thereby Suppressing the Pathway
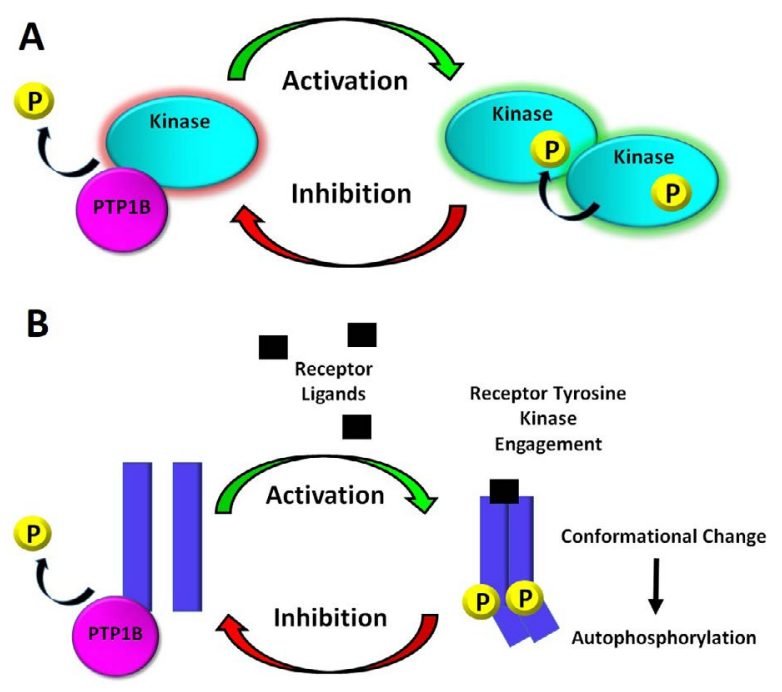

Figure 2: Phosphatases Keep Activity of Kinases in Check by Catalysing Remova of Critical Phosphate Groups to Dampen Activity, Thereby Suppressing the Pathway, and Target Both Non-Receptor Kinases (A) and Receptor Kinases (B). Pharmacological Inhibition of Phosphatases Relieves Inhibition of the Kinase Thereby Enhancing Kinase Activity and Upregulating Downstream Signaling Cascades
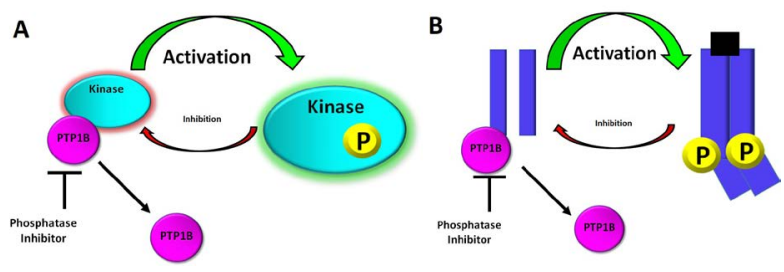

Much effort has gone into identifying kinase inhibitors to blunt pathways that are upregulated in the disease state. Conversely, upregulating alternate kinase-dependent pathways that are suppressed in a disease state might prove to be advantageous. Identifying mechanisms to upregulate the latter has become a more recent focus, with phosphatases presenting a promising target to do so. Phosphatases keep activity of kinases in check by catalysing removal of critical phosphate groups to dampen activity, thereby suppressing the pathway. Thus, phosphatases are inhibitors of kinases and inhibiting the inhibitors provides an approach to harness endogenous processes to stimulate activity of relevant kinases.

\section{PTP1B AS A THERAPEUTIC TARGET IN VASCULAR DISEASE -}

Activation of protein-tyrosine phosphatase 1B (PTP1B) has been shown to reduce signaling through insulin and leptin-dependent pathways- by dephosphorylating and switching off the pathway and has therefore received interest as a potential therapeutic target to upregulate insulin and leptin signaling. Leptin influences appetite and energy expenditure while insulin controls glucose uptake and glycogen synthesis, through a tyrosine kinase receptor regulated by PTP1B. Enhancing activity (by inhibiting PTP1B) would, therefore, be predicted to permit these signaling pathways to persist, resulting in suppressed appetite and enhancing glucose uptake and glycogen synthesis. This is particularly relevant in treating diabetes and obesity. Insulin resistance is both a prominent and causative feature in these settings, and enhancing insulin sensitivity would improve metabolic parameters. Indeed, as a negative regulator of both leptin and insulin signaling, inhibition of PTP1B has been validated as a relevant therapeutic approach. Deletion of the PT$\mathrm{P} 1 \mathrm{~B}$ gene in mice resulted in increased insulin and leptin sensitivity and resistance to obesity induced by a high-fat diet (reviewed in). ${ }^{2}$

Pharmacological inhibition using a small molecule inhibitor of PTP1B, trodusquemine (MSI-1436) has been tested over the last 10 years in completed Phase I trials for obesity and diabetes (https://clinicaltrials.gov/ ID NCT00509132, NCT00606112, NCT00806338, Genaera Corporation) and in breast cancer (NCT02524951). The latter was terminated however this year due to lack of sponsorship and results from completed trials have not been formally published. Nonetheless, Genaera Corporation presented their findings at two conferences and a summary can be found online, indicating that no adverse events were observed and that the drug was well tolerated. ${ }^{3,4}$ A small-scale ( $\left.n=92\right)$ Phase II trial in diabetes is now complete in which inhibition of PTP1Bwas achieved by downregulation using antisense technology (IONISPTP-1BRx, Ionis Pharmaceuticals, https://clinicaltrials.gov/ ID NCT01918865). The results published this year showed that in combination with metformin alone or with a sulfonylurea, IONISPTP-1BRx improved glycaemic parameters and suppressed leptin signaling. ${ }^{5}$

Trials so far relate to treatment of diabetes and breast cancer however evidence exists to suggest that PTP1B inhibition might also be relevant in reducing risk of myocardial infarction (MI) through suppressing the development of atherosclerotic plaques. Inhibition of PTP1B using MSI-1436 was performed in a mouse model of atherosclerosis (low-density lipoprotein receptor null (LDLR) mice on a high-fat diet). The authors found that MSI1436 treatment reduced plaque burden at the conclusion of the experiment and suggested that PTP1B inhibition both prevents and reverses plaque formation. ${ }^{6}$ It has been highlighted, however that reversal of existing plaques was not definitively demonstrated, although PTP1B inhibition might certainly retard development. ${ }^{7}$ The authors were unable to demonstrate PTP1B inhibition specifically, however but knocking out PTP1B in macrophages suppressed plaque development in an alternative mouse model of atherosclerosis (ApoE deficient mice). ${ }^{8}$ These studies together suggest that PTP1B inhibition might reduce the risk of $\mathrm{MI}$ in patients with atherosclerosis by slowing plaque progression.

While results for MSI-1436 in current pre-clinical studies and clinical trials are encouraging, a drawback of this molecule is that it is not orally bioavailable. Drug delivery is achieved by injec- 
tion which is not ideal for prolonged treatment from the patient perspective. Of note, an orally bioavailable analog DPM-1001 has been described this year. ${ }^{9}$ In addition to improved drug delivery properties, DPM-1001 is a more potent inhibitor of PTP1B, with an IC50 of $100 \mathrm{nM}$ rather than $600 \mathrm{nM}$ by MSI-1436. ${ }^{9}$ In preclinical models of obesity (diet-induced), similarly to MSI-1436, DPM-1001 marginally reduced weight over 3-weeks (by $5 \%$ ) in obese mice. Concomitantly, glucose was cleared more quickly from the bloodstream and insulin sensitivity (exogenously administered insulin-induced glucose uptake) was increased. Phosphorylation/ activation of downstream targets of leptin and insulin (JAK2 and insulin receptor respectively) was also increased, supporting enhanced leptin and insulin signaling as being the mechanism responsible, similarly to MSI-1436. ${ }^{9}$ Thus inhibition of PTP1B was able to upregulate critical kinase-dependent pathways to improve outcomes in diabetic and obesity models.

The same authors turned their attention to developing another small molecular inhibitor of PTP1B that would act through an alternative mechanism to MSI-1436 and DPM-1001. ${ }^{10}$ Insulin signaling triggers localized production of reactive oxygen species that transiently oxidize and induce conformational changes in PTP1B to suppress its activity. This therefore optimally activates signaling through insulin receptor engagement and is a reversible reaction, whereby reduction restores the activity of PTP1B. Chelerythrine, formerly identified as a PKC inhibitor, binds directly and selectively to the oxidized inactive conformation of PTP1B and stabilizes this structure. By inhibiting PTP1B, Chelerythrine was able to enhance insulin and leptin signaling pathways. Similar to MSI-1436 and DPM-1001, in a mouse model of obesity (diet-induced) chelerythrine induced mild (3\%) weight loss and improved both glucose tolerance and insulin sensitivity. ${ }^{10}$

\section{PTP1B AS A THERAPEUTIC TARGET IN THE HEART}

These approaches may also prove to be relevant to treating patients following myocardial infarction (MI). PTP1B activity is increased in failing hearts in the clinic $^{11}$ and inhibition either by pharmacological or genetic means improves heart function after MI. ${ }^{12-14}$ PTP1B null mice were protected against cardiac dysfunction following $\mathrm{MI}^{12,13}$ with similar results obtained by pharmacological inhibition using another pharmacological inhibitor AS279. ${ }^{12}$ While infarct size was unchanged, fibrosis was reduced and cardiac function enhanced. ${ }^{12}$ In the null model, it was shown that angiogenesis was enhanced by PTP1B inhibition in MI. ${ }^{13}$ In the context of the inhibitor MSI-1436 that has not shown any adverse events in Phase I trials, MSI-1436 improved outcomes in two animal models of cardiac injury. ${ }^{14}$ The zebrafish is capable of regenerating following resection injury (removal of a portion of the tissue) in both the caudal (tail) fin and the heart. ${ }^{15,16}$ In both models, MSI-1436 enhanced the rate of regeneration and cellular proliferation by 2-3fold. ${ }^{14}$ Importantly, aberrant overgrowth or tissue malformation was not observed in restored tissues nor was MSI-1436 toxic to zebrafish embryos or uninjured adult animals. ${ }^{14}$ Similarly, in mice in subjected to MI, heart function improved by 2 -fold concomitant with a $53 \%$ reduction in infarct size, reduced progression to cardiac dilation and heart failure and increased cellular proliferation in the border zone surrounding the infarcted area. ${ }^{14}$ It should be noted, however that modulating contractile function through PTP1B inhibition in settings such as hypertrophic cardiomyopathy (HCM) might prove to be deleterious ${ }^{17,18}$ although positive results have also been observed. ${ }^{19,20}$ Whether this is a valid approach in HCM therefore, is unclear. Nonetheless, in the context of cardiac injury PTP1B inhibition has shown promising results. ${ }^{12-14}$

In terms of the mechanisms involved, PTP1B has been shown to regulate a multitude of pathways relevant to cardiac function and disease including metabolism, cell cycle, and cell survival. ${ }^{21}$ In the heart specifically, PTP1B has been suggested to play an important role in regulating contractile function (reviewed in $^{22}$ ) which may at least in part, be due to the regulation of insulin signaling which itself is blunted in the failing heart concomitantly with enhanced PTP1B activity. ${ }^{11}$ Some mechanisms that are directly implicated therefore include insulin-mediated metabolic regulation and cardioprotection, ${ }^{11,23}$ increased tissue perfusion through enhanced angiogenesis, ${ }^{13}$ endothelial protection which may be nitric oxide dependent ${ }^{6,13,24}$ and improved contractile function. ${ }^{22}$ Insulin- independent mechanisms also exist, and indeed, PTP1B has many other potential targets. MSI-1436 administration in atherosclerotic mice resulted in upregulation of Akt-AMPK $\alpha 1$ with no effect on insulin receptor activation. ${ }^{6}$ Here, MSI-1436 was able to protect against plaque formation potentially through reduction of serum lipid levels and/or suppressing systemic inflammation ${ }^{6,8}$ although the authors could not find any evidence of PTP1B inhibition itself. ${ }^{6}$ This could reflect sub-optimal detection conditions but MSI-1436 may also have off-target effects which have not yet been identified. Any number of combinations of the above might be responsible for the mechanisms by which PTP1B inhibitors act. Further, a number of cell types might be targeted in which alternative PTP1B regulated pathways play a critical role including immune cells, progenitor cells, endothelium, smooth muscle cells, fibroblasts, and cardiomyocytes. Given the ubiquitous expression of PTP1B and the plethora of targets, likely a combination of pathways and/or cell types are modulated by inhibiting PTP1B.

In summary, activation of PTP1B has been observed in the injured and/or failing heart both in mouse models and in humans, and its inhibition suppresses progression to heart failure. While no pharmacological inhibitors have yet been tested clinically for this indication, early trials in others have shown promising results and the next generation of inhibitors is under development. There is therefore great potential for PTP1B inhibitors to be applicable in the setting of $\mathrm{MI}$ in the near future.

\section{REFERENCES}

1. Fiedler LR, Maifoshie E, Schneider MD. Mouse models of heart failure: cell signaling and cell survival. Curr Top Dev Biol. 2014; 109: 171-247. doi: 10.1016/B978-0-12-397920-9.00002-0

2. Tsou RC, Bence KK. The Genetics of PTPN1 and Obesity: Insights from mouse models of tissue-specific PTP1B deficiency. $J$ Obes. 2012. doi: 10.1155/2012/926857

3. Genaera Corporation. GENAERA : Presents Preliminary Phase 1b Data for Trodusquemine (MSI-1436). Website: https:// 
www.marketscreener.com/GENAERA-9416/news / GENAERA-Presents-Preliminary-Phase-1b-Data-for-TrodusquemineMSI-1436-13123592/. Accessed November 10, 2018.

4. Bio Space. Genaera Corporation Presents Preliminary Phase 1b Data for Trodusquemine (MSI-1436) . Website: https://www.biospace.com/article/releases/genaera-corporation-presents-phase1-data-for-trodusquemine-msi-1436-/. Accessed November 10, 2018.

5. Digenio A, Pham NC, Watts LM et al. Antisense inhibition of protein tyrosine phosphatase $1 \mathrm{~B}$ with IONIS-PTP-1B ${ }_{\mathrm{Rx}}$ improves insulin sensitivity and reduces weight in overweight patients with type 2 diabetes. Diabetes Care. 2018; 41(4): 807-814. doi: 10.2337/ dc17-2132

6. Thompson D, Morrice N, Grant L, et al. Pharmacological inhibition of protein tyrosine phosphatase $1 \mathrm{~B}$ protects against atherosclerotic plaque formation in the LDLR-/- mouse model of atherosclerosis. Clin Sci (Lond). 2017; 131(20): 2489-2501. doi: 10.1042/CS20171066

7. Moxon JV, Moran CS, Golledge J. Comment on 'Pharmacological inhibition of protein tyrosine phosphatase $1 \mathrm{~B}$ protects against atherosclerotic plaque formation in the LDLR-/- mouse model of atherosclerosis'. Clin Sci (Lond). 2018; 132 (1): 37-38. doi: 10.1042/CS20171522

8. Thompson D, Morrice N, Grant L, et al. Myeloid protein tyrosine phosphatase 1B (PTP1B) deficiency protects against atherosclerotic plaque formation in the ApoE $\mathrm{E}^{-/-}$mouse model of atherosclerosis with alterations in IL10/AMPK $\alpha$ pathway. Mol Metab. 2017; 6(8): 845-853. doi: 10.1016/j.molmet.2017.06.003

9. Krishnan N, Konidaris KF, Gasser G, Tonks NK. A potent, selective, and orally bioavailable inhibitor of the protein-tyrosine phosphatase PTP1B improves insulin and leptin signaling in animal models. J Biol Chem. 2018; 293(5): 1517-1525. doi: 10.1074/jbc. C117.819110

10. Krishnan N, Bonham CA, Rus IA, et al. Harnessing insulin- and leptin-induced oxidation of PTP1B for therapeutic development. Nat Commun. 2018; 9(1): 283. doi: 10.1038/s41467-017-02252-2

11. Nguyen TD, Schwarzer M, Schrepper A, et al. Increased protein tyrosine phosphatase 1B (PTP1B) activity and cardiac insulin resistance precede mitochondrial and contractile dysfunction in pressure-overloaded hearts. J Am Heart Assoc. 2018; 7(13). doi: 10.1161/JAHA.118.008865

12. Gomez E, Vercauteren M, Kurtz B et al. Reduction of heart failure by pharmacological inhibition or gene deletion of protein tyrosine phosphatase 1B. J Mol Cell Cardiol. 2012; 52(6): 1257-1264. doi: 10.1016/j.yjmcc.2012.03.003
13. Besnier M, Galaup A, Nicol L et al. Enhanced angiogenesis and increased cardiac perfusion after myocardial infarction in protein tyrosine phosphatase 1B-deficient mice. FASEB J. 2014 ; 28(8): 3351-3361. doi: 10.1096/fj.13-245753

14. Smith AM, Maguire-Nguyen KK, Rando TA, et al. The protein tyrosine phosphatase 1B inhibitor MSI-1436 stimulates regeneration of heart and multiple other tissues. NPJ Regen Med. 2017; 2: 4. doi: 10.1038/s41536-017-0008-1

15. Poss KD, Wilson LG, Keating MT. Heart regeneration in zebrafish. Science. 2002; 298(5601): 2188-2190. doi: 10.1126/science. 1077857

16. Nakatani Y, Kawakami A, Kudo A. Cellular and molecular processes of regeneration, with special emphasis on fish fins. Dev Growth Differ. 2007; 49(2): 145-154. doi: 10.1111/j.1440169X.2007.00917.x

17. Grote-Wessels S, Baba HA, Boknik P, et al. Inhibition of protein phosphatase 1 by inhibitor-2 exacerbates progression of cardiac failure in a model with pressure overload. Cardiovasc Res. 2008; 79: 464-471. doi: 10.1093/cvr/cvn113

18. El-Armouche A, Wittkopper K, Degenhardt F, et al. Phosphatase inhibitor-1-deficient mice are protected from catecholamineinduced arrhythmias and myocardial hypertrophy. Cardiovasc Res. 2008; 80: 396-406. doi: 10.1093/cvr/cvn208

19. Pathak A, del Monte F, Zhao W, et al. Enhancement of cardiac function and suppression of heart failure progression by inhibition of protein phosphatase 1. Circ Res. 2005; 96: 756-766. doi: 10.1161/01.RES.0000161256.85833.fa

20. Yamada M, Ikeda Y, Yano M, et al. Inhibition of protein phosphatase 1 by inhibitor-2 gene delivery ameliorates heart failure progression in genetic cardiomyopathy. FASEB J. 2006; 20(8): $1197-$ 1199. doi: 10.1096/fj.05-5299fje

21. Ceulemans H, Bollen M. Functional diversity of protein phosphatase-1, a cellular economizer and reset button. Physiol Rev. 2004; 84(1): 1-39. doi: 10.1152/ physrev.00013.2003

22. Biesiadecki J, Ziolo MT. Should we treat heart failure with phosphatase inhibitors? Better to start at the end. J Mol Cell Cardiol. 2015; 89: 116-118. doi: 10.1016/j.yjmcc.2015.10.020

23. Fu F, Zhao K, Li J, et al. Direct evidence that myocardial insulin resistance following myocardial ischemia contributes to post-ischemic heart failure. Sci Rep. 2015; 5: 17927. doi: 10.1038/srep17927

24. Vercauteren M, Remy E, Devaux C, et al. Improvement of peripheral endothelial dysfunction by protein tyrosine phosphatase inhibitors in heart failure. Circulation. 2006; 114(23): 2498-2507. doi: 10.1161/CIRCULATIONAHA.106.630129 